\title{
Distinguishing between similar miniproteins with single-molecule nanopore sensing: a
}

\section{computational study}

\author{
Sebastian Cardoch, ${ }^{* \dagger}$ Nicusor Timneanu, ${ }^{\dagger}$ Carl Caleman, ${ }^{\ddagger}, \dagger$ and Ralph $\mathrm{H}$. \\ Scheicher ${ }^{*} \dagger$ \\ $\dagger$ Department of Physics and Astronomy, Uppsala University, Box 516, SE-751 20, Uppsala, \\ Sweden \\ $\ddagger$ Center for Free-Electron Laser Science, Deutsches Elektronen Synchrotron DESY, \\ Notkestraße 85, 22607 Hamburg, Germany \\ E-mail: sebastian.cardoch@physics.uu.se; ralph.scheicher@physics.uu.se
}

\section{Supporting Information Available}

\section{MD simulation results}

Additional results for the miniproteins' center of mass and number of ions inside the pore as a function of time are shown in figures 1 and 2, respectively. From the last 20 ns of simulation, we calculated the orientation of the miniproteins' dipole moment from its center of mass. In figure 3 we show the time-evolution of the angle $\phi$ made between the dipole vector and the + z-axis. We considered the miniprotein aligned with the external field when its dipole moment was at an angle $\leq 50^{\circ}$ with the z-axis. Based on the alignment, we generated normalized histograms of the LPF ionic current. These results are shown in figure 4. 

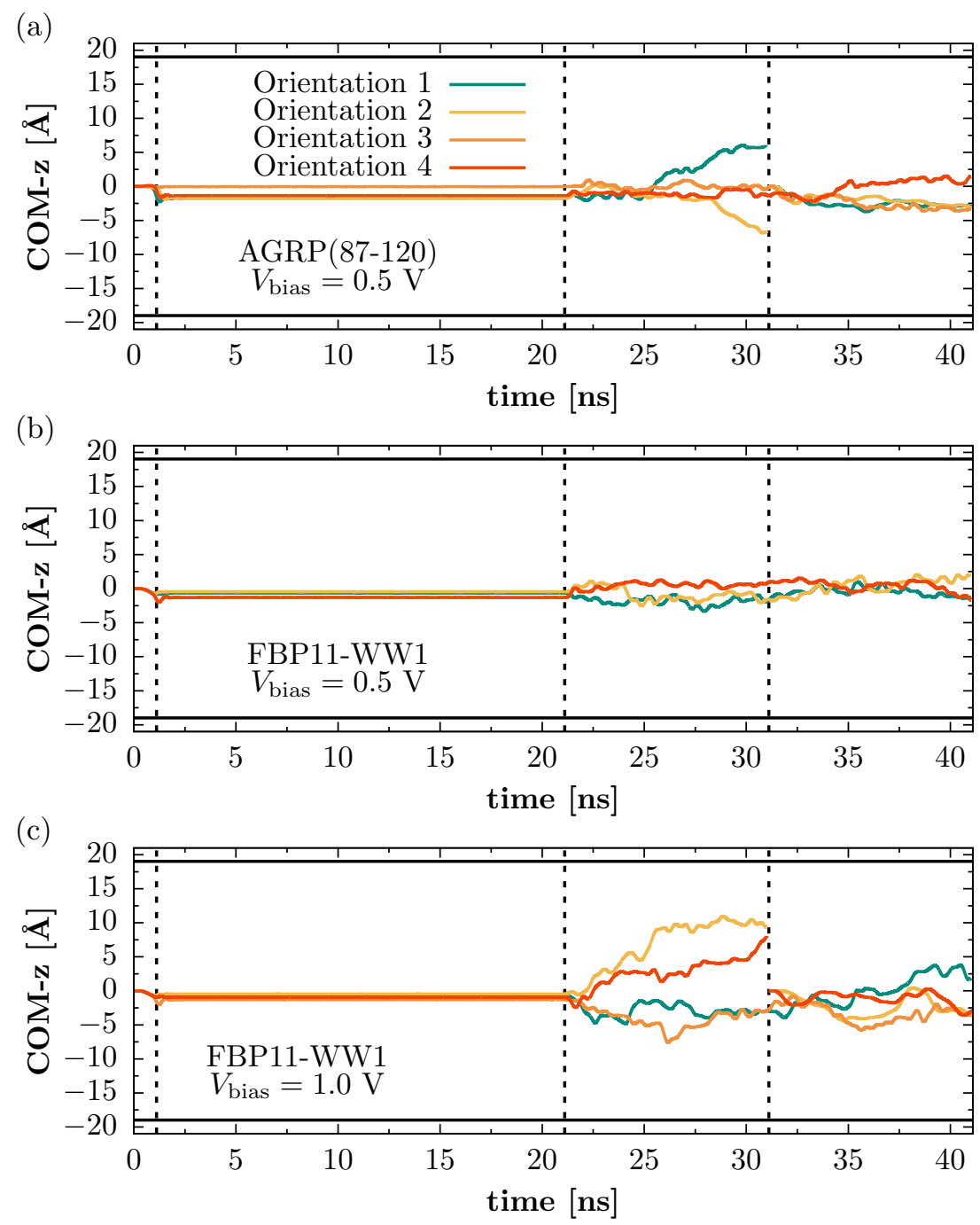

Figure 1: COM z-direction for miniproteins as a function of the simulation time. The results have been smoothed out with a LPF using a $1 \mathrm{GHz}$ cutoff frequency. 

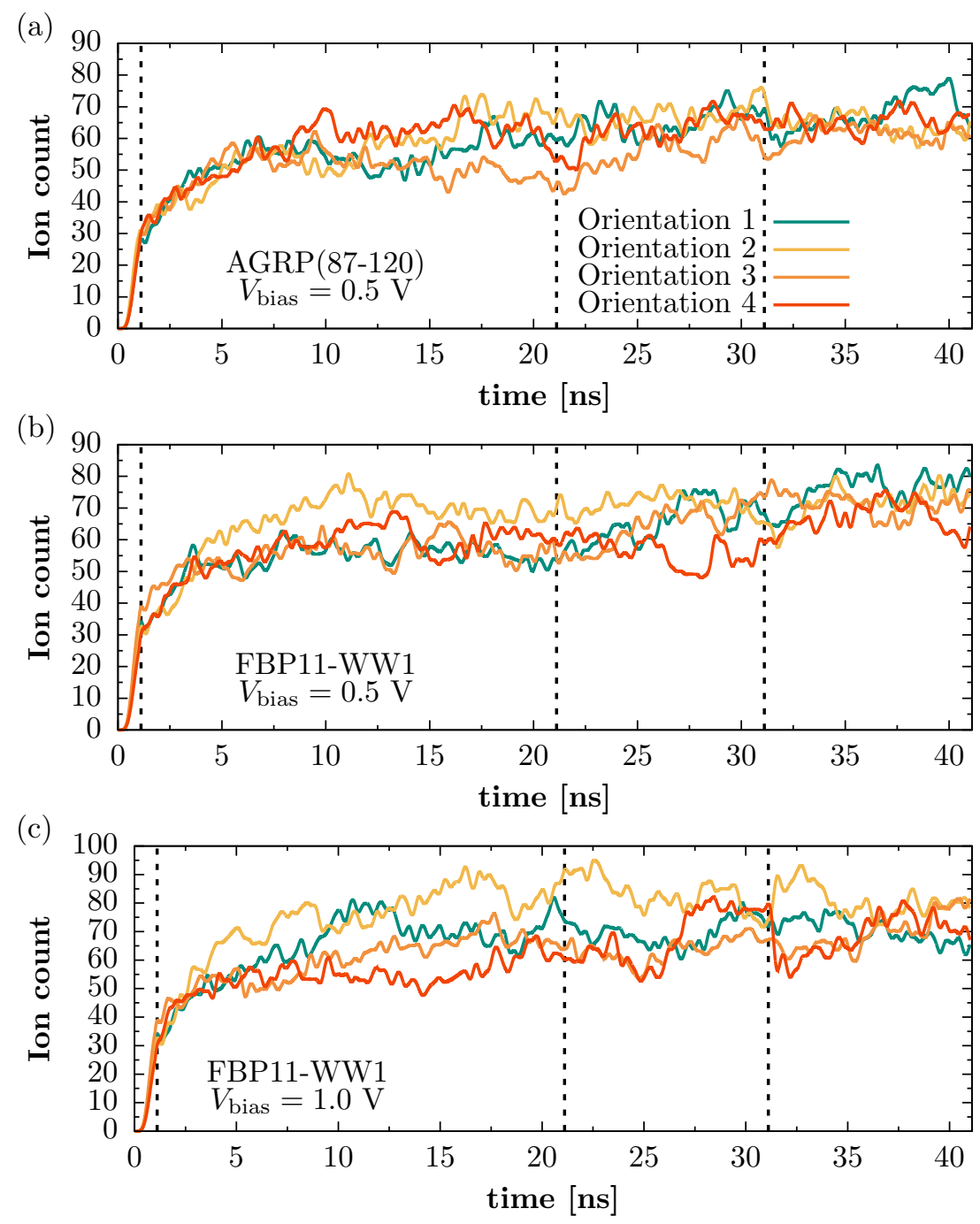

Figure 2: The instantaneous number of ions inside the occupied pore as a function of simulation time. The results have been smoothed out with a LPF using a $1 \mathrm{GHz}$ cutoff frequency. 


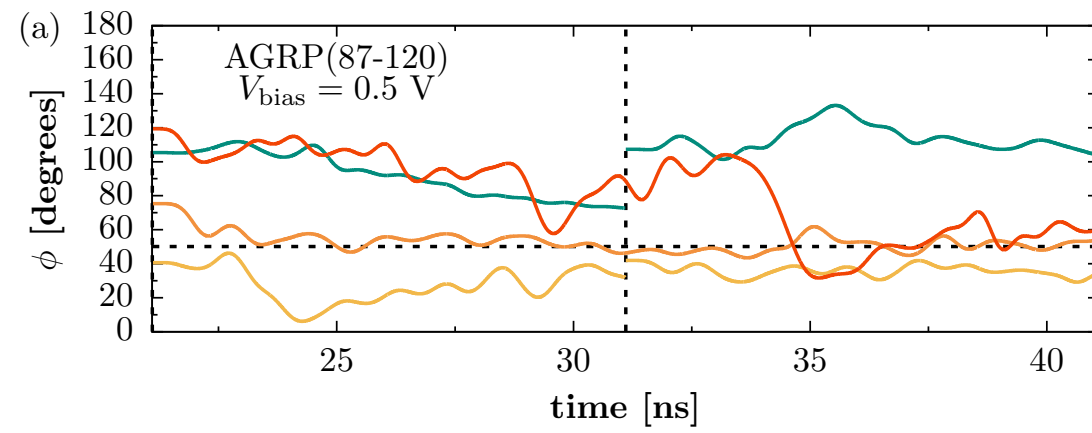

Orient. 1

Orient. 2

Orient. 3

Orient. 4
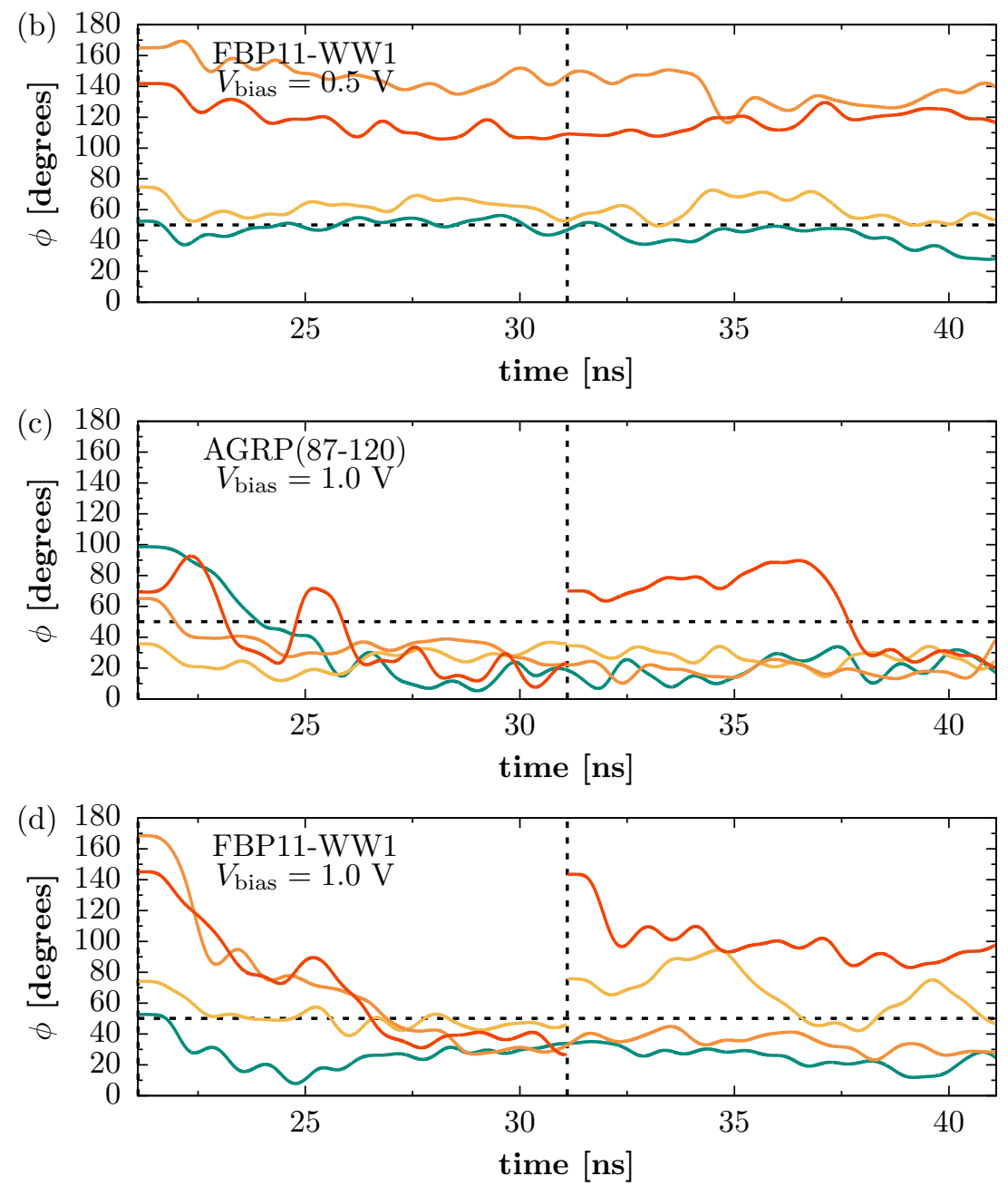

Figure 3: Miniprotein dipole moment direction as a function of time. The dipole direction was determined by the angle $\phi$ made between the dipole vector and the z-axis along the positive direction. Full alignment with the external field happens at $0^{\circ}$ and anti-alignment happens at $180^{\circ}$. 

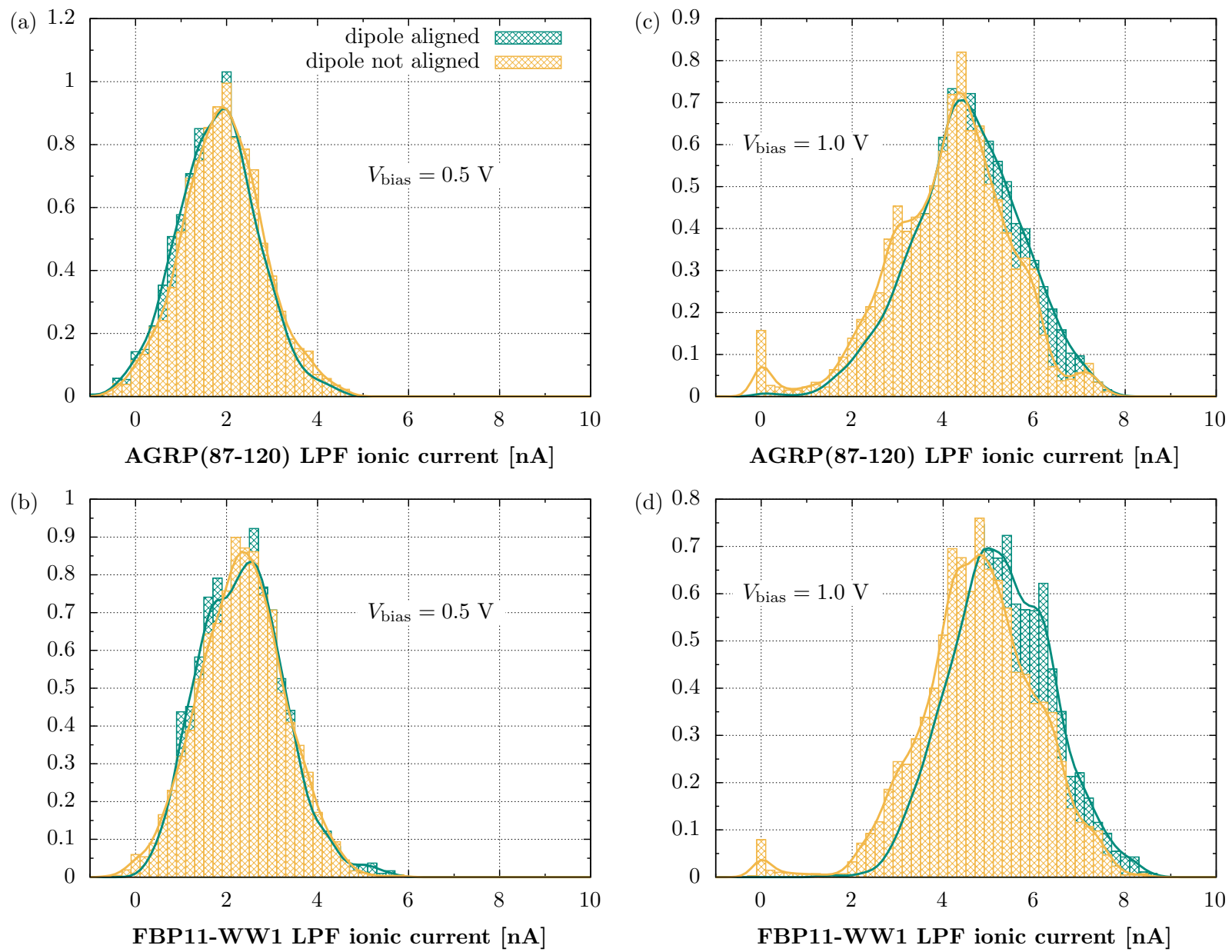

Figure 4: Comparing LPF ionic current histograms for when miniproteins are aligned or not aligned with the external field. To compare these two scenarios, histograms are normalized to the number of data points available for each distribution. 


\section{Miniprotein information}

Information about the two miniproteins investigated is shown in table 1 . Identity is a property that measures the number of amino acids that, excluding gaps, exactly match in both sequences. Figure 5 shows the optimal alignment of the two miniprotein sequences, where we find an identity value of $17.39 \%$. The identity indicates similarities in the primary structure of the two miniproteins and shows that their sequence differs significantly.

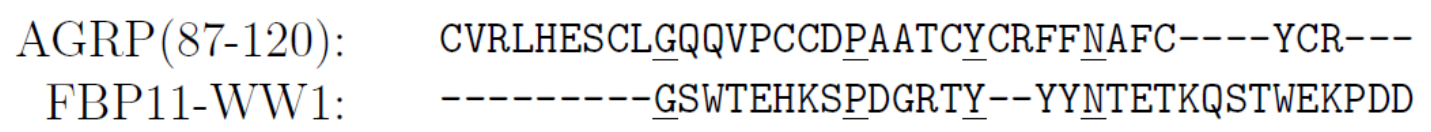

Figure 5: Aligned miniprotein sequences, with amino acids represented by their single-letter residue codes and gaps by a dash symbol. Identity value and the aligned sequence were obtained with Clustal Omega ${ }^{1}$.

Table 1: Selected miniproteins. Their structure has been determined by NMR spectroscopy in an aqueous environment, and their files were obtained from the Protein Data Bank $(\mathrm{PDB})^{2}$.

\begin{tabular}{llrrrr}
\hline PDB & name & length $^{a}(\AA)$ & mass $(\mathrm{Da})$ & volume $^{b}\left(\AA^{3}\right)$ & residues \\
\hline 1ZR7 & FBP11 WW1 & 30.7 & 3587.7 & $8.47 \times 10^{3}$ & 30 \\
1MR0 & AGRP $(87-120)$ & 31.6 & 3884.5 & $8.90 \times 10^{3}$ & 34 \\
\hline
\end{tabular}

${ }^{a}$ Largest distance between any two atomic centers; ${ }^{b}$ Approximate volume determined using MDTools MolVolume with a probe radius of $2.0 \AA$ and grid size of $0.1 \AA$.

\section{Relative permittivity silicon nitride}

To compute the permittivity we ran molecular dynamics simulations on a silicon nitride sphere. The sphere had a radius of $36.5 \AA$ and was placed in vacuum inside a simulation cube $180 \AA$ in length and held at a constant temperature of $298 \mathrm{~K}$ using a Lowe-Andersen thermostat. The relative permittivity $\epsilon_{r}$ was computed from

$$
E_{z}=\frac{3}{2+\epsilon_{\mathrm{r}}} E_{\mathrm{ext}}
$$


where $E_{\text {ext }}$ is the field produced by the bias voltage applied along the z-direction and $E_{z}$ is the field inside the sphere. We assumed the sphere had a uniform charge density such that the electric field inside depended only on its surface charge density. Inside the sphere, the field was computed using

$$
E_{z}=-\frac{1}{3 \epsilon_{0}} P_{z},
$$

where $P_{z}$ is the polarization and $\epsilon_{0}$ is the permittivity of free space. The polarization was computed from the time-averaged dipole moment change and time-averaged volume using

$$
P_{z}=\frac{\left\langle\Delta D_{z}\right\rangle_{t}}{\langle V\rangle_{t}} .
$$

We explored $\epsilon_{r}$ at different harmonic restraints and bias voltages, with results shown in figure 6 .
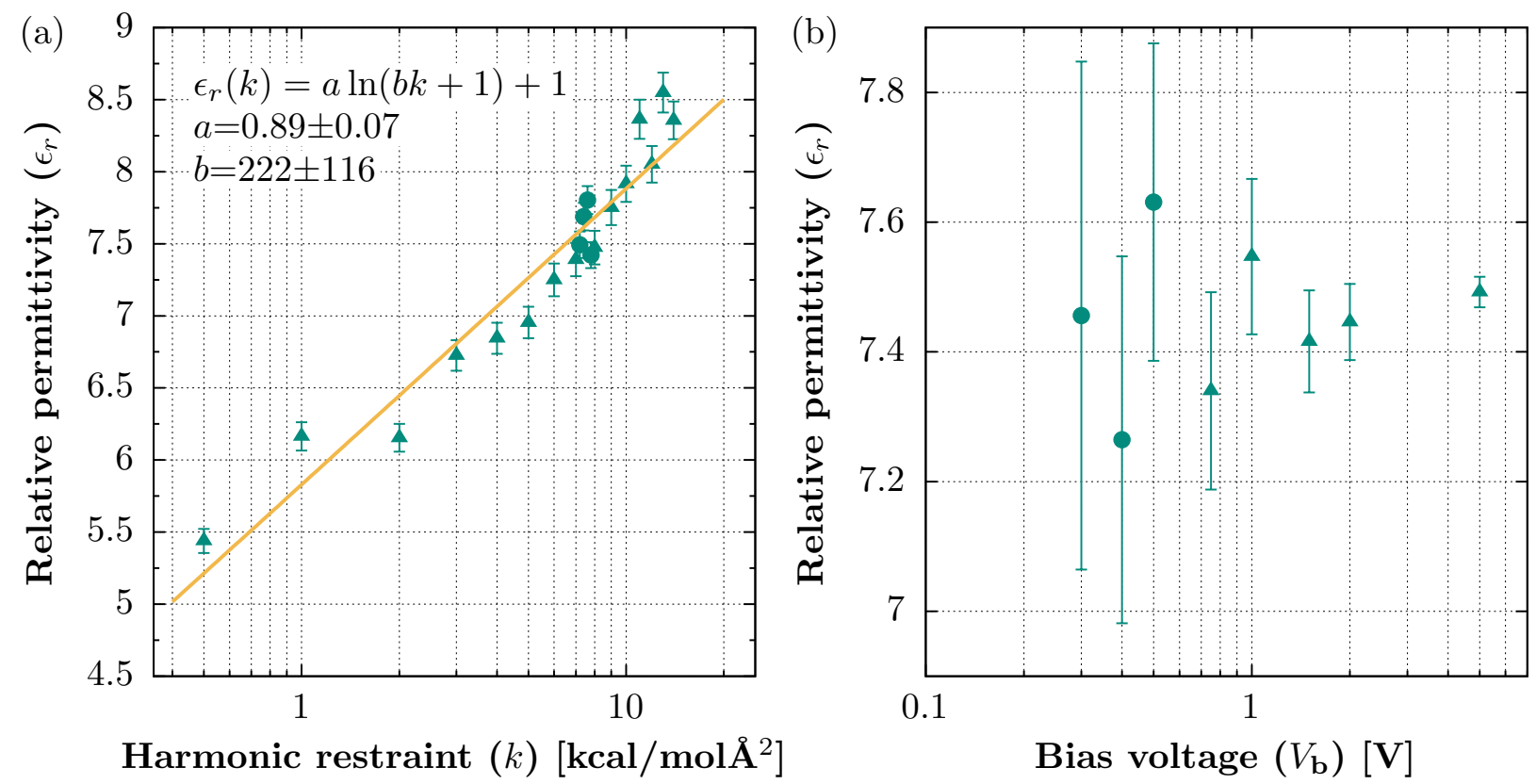

Figure 6: (a) Relative permittivity as a function of harmonic restraints. Triangles represent values obtained from 1.0 ns-long simulations and circles from 1.6 ns-long simulations. We fitted the results to a logarithmic weighted best fit. (b) Relative permittivity for different applied bias voltages. Values represented by triangles and circles were obtained from $1 \mathrm{~ns}$ and 2 ns-long simulations, respectively. Uncertainties in (a) and (b) are derived from fluctuations in the computed time-averaged dipole moment and sphere's volume. 


\section{Pore surface silicon-nitrogen ratio}

All atoms in the silicon nitride membrane, except those near the pore surface, were given a harmonic restraint of $1 \mathrm{kcal} /\left(\operatorname{mol} \AA^{2}\right)$. The system was placed in vacuum, minimized for $0.01 \mathrm{~ns}$, equilibrated for $0.15 \mathrm{~ns}$ at $298 \mathrm{~K}$ using a Lowe Andersen thermostat, and again minimized for $0.01 \mathrm{~ns}$. Surface silicon and nitrogen counts were based on trajectory files stored $0.1 \mathrm{ps}$, with results shown in figure 7 .

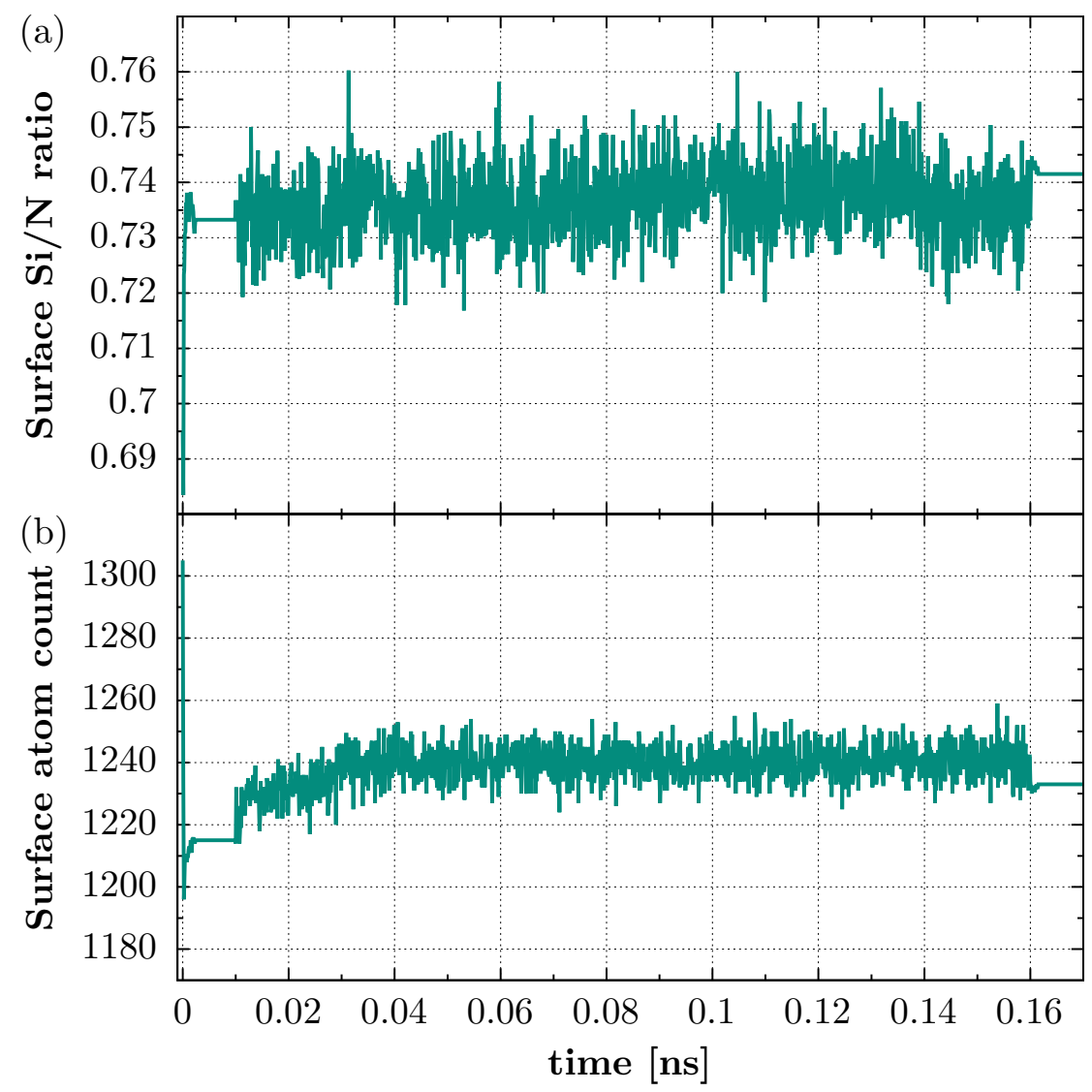

(c)

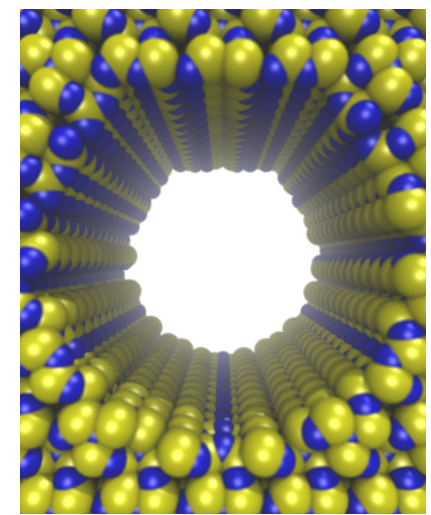

(d)

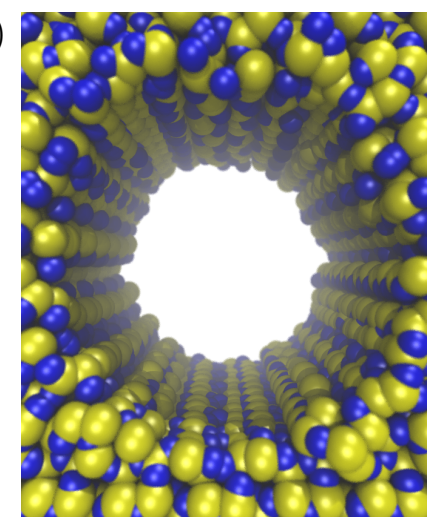

Figure 7: (a) Silicon-nitrogen atom ratio at the surface of the nanopore. (b) The number of atoms being considered as surface atoms in the calculation of the silicon/nitrogen ratio. (c)(d) Before and after snapshots of the pore surface atomic arrangement.

\section{Ion conductivity}

The Lowe-Andersen thermostat keeps a target temperature by modifying the velocity of pairs of particles within a search radius $R_{T}$, where each pair has a probability $\Gamma \Delta t$ of colliding 
with the heat bath ${ }^{3,4}$. Thermostat applies a shear stress that contributes with an artificial viscosity

$$
\eta^{T} \propto \frac{\pi \rho^{2} R_{T}^{5} \Gamma}{75 m}
$$

where $m$ and $\rho$ in our case are the mass and density of water, $\Gamma$ is the collision rate and $R_{T}$ the search radius ${ }^{3,4}$. To adjust the ion conductivity in MD simulations, we benefited from $\eta^{T}$. In principle, parameters $R_{T}$ and $\Gamma$ can be modified, but it is desirable to have a large collision rate that keeps the temperature constant and achieves a high rate of diffusion ${ }^{3}$.

We based our ion conductivity calibration on methods presented in Aksimentiev and Schulten ${ }^{5}$. The reference molar conductivity $\kappa=14.985 \mathrm{~S} / \mathrm{m}$ for potassium chloride was determined from textbook ionic molar conductivities in bulk solution ${ }^{6}$. We simulated a cube of water $4 \mathrm{~nm}$ in length with a $1 \mathrm{M}$ concentration of $\mathrm{KCl}$ using target temperature $298 \mathrm{~K}$. For a fixed $R_{T}$ value and after a $0.05 \mathrm{~ns}$ equilibration (NPT conditions with $1 \mathrm{~atm}$ ), we simulated the system for $1.0 \mathrm{~ns}$ (NVT conditions) under a range of $0.1-5.0 \mathrm{~V}$ bias voltages. Results from the simulations are shown in figure 8.

\section{Energy convergence}

We were interested in mesh cutoff and cell dimension parameters that gave a well-converged ground state energy for silicon nitride and amino acid in vacuum. We examined these parameters for two systems: (1) amino acid glutamine with 20 atoms in total and (2) silicon nitride together with glutamine with 300 atoms in total. We employed a double- $\zeta$ polarized basis set with the van der Waals functional DRSLL. Convergence was achieved using Pulay with a mixing factor of 0.1 for system (1) and 0.02 for system (2), and a history of 5 selfconsistent cycles. The convergence results are shown in figure 9 . 

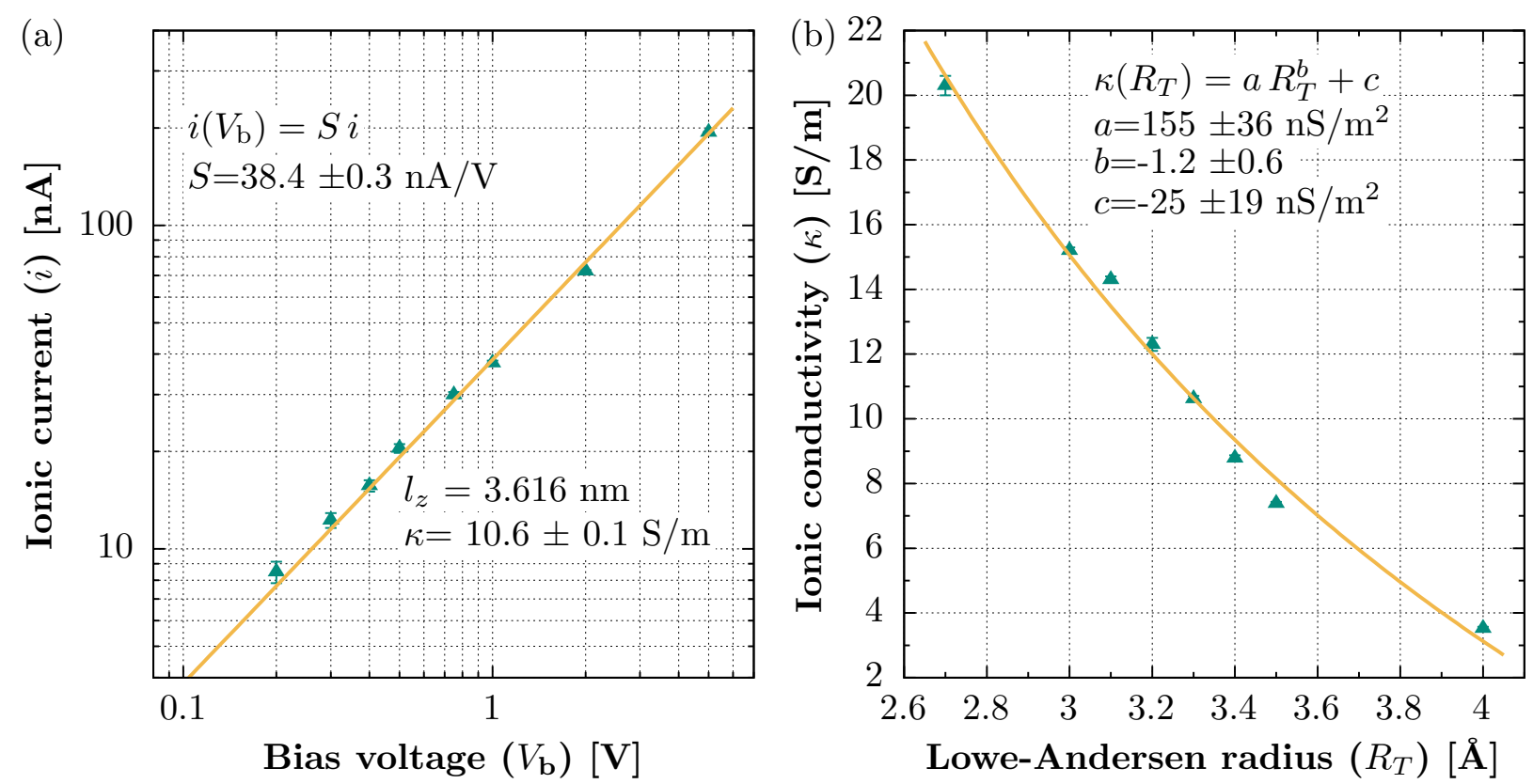

Figure 8: (a) Ionic current as a function of applied bias voltage for a fixed Lowe-Andersen radius $R_{T}=3.0 \AA$. The ionic current was computed over the entire length of the simulation box, using ions' trajectories stored every $0.1 \mathrm{ps.} \mathrm{The} \mathrm{slope} \mathrm{of} \mathrm{the} \mathrm{best} \mathrm{fit} \mathrm{is} \mathrm{the} \mathrm{conductance}$ $S$. (b) Ion conductivity for a range of $R_{T}$ values, based on the slope of the weighted linear best fit of the current vs. bias voltage plots.

\section{Terminal velocity}

For the dwell time, we determined the constant $A$ based on the protein translocation without interaction with the pore surface. We imagined the protein experiences some acceleration subject to the electric field $F_{e}$ and drag force $F_{d}$

$$
m a=F_{\mathrm{e}}-F_{d}
$$

From MD simulations, we estimated that the leading contribution from the drag force happens in the limit of small velocity $(\mathcal{O}(0.1 \mathrm{~m} / \mathrm{s})$ for $\Delta d=2 \mathrm{~nm}, t=10 \mathrm{~ns})$. The miniprotein moves through a viscous fluid and the drag force is proportional to the velocity. This effect is shown in figure 10. The net force can be written as

$$
\frac{d v}{d t}=a_{e}-k_{d} v
$$


(a)

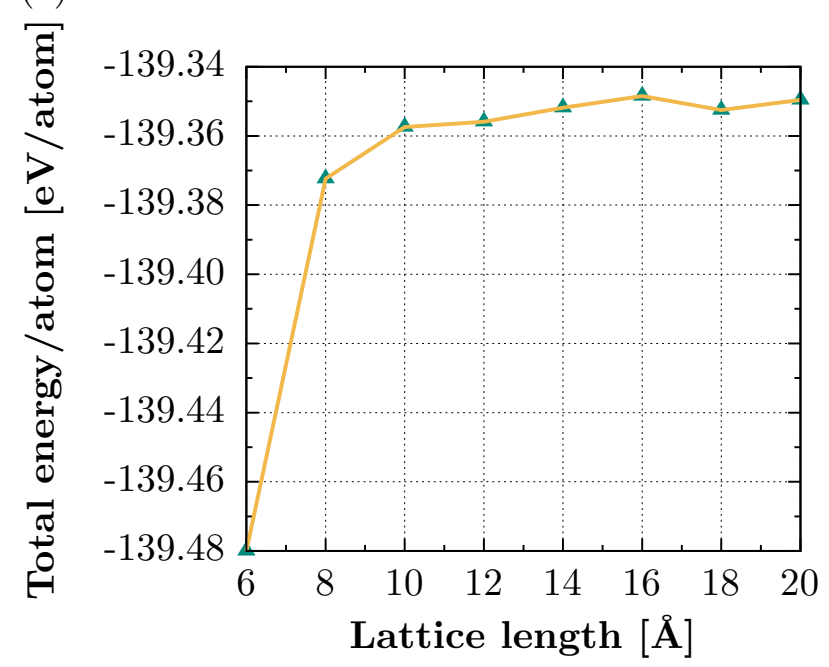

(c)

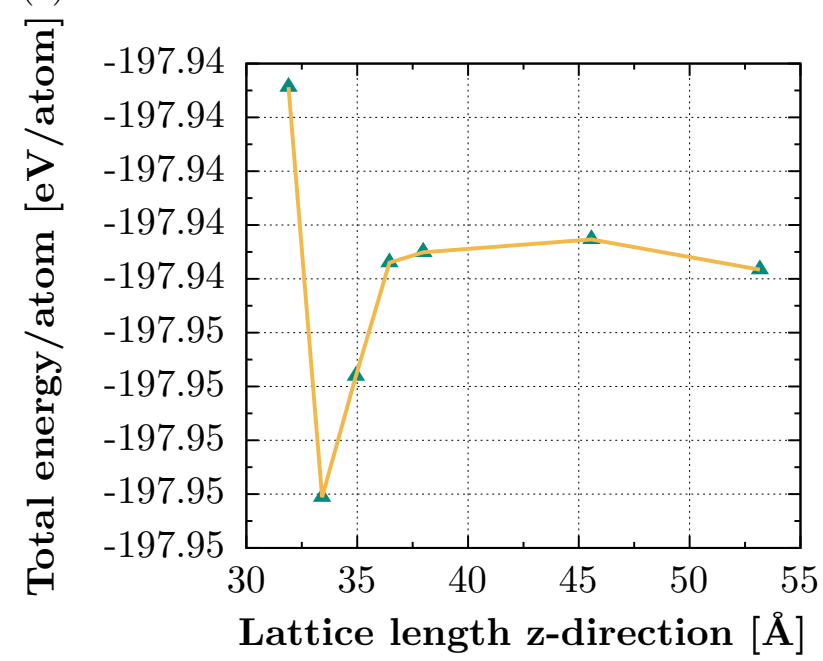

(b)

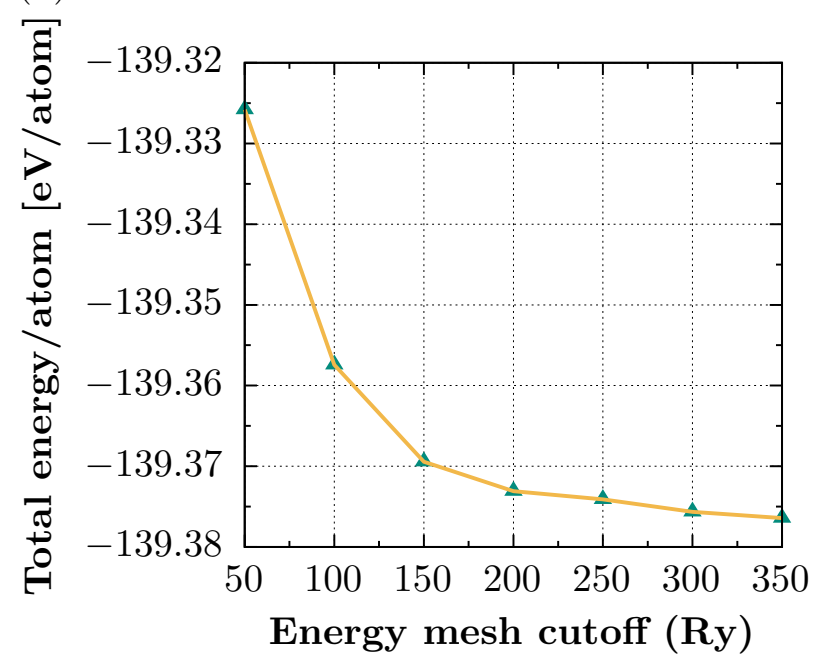

(d)

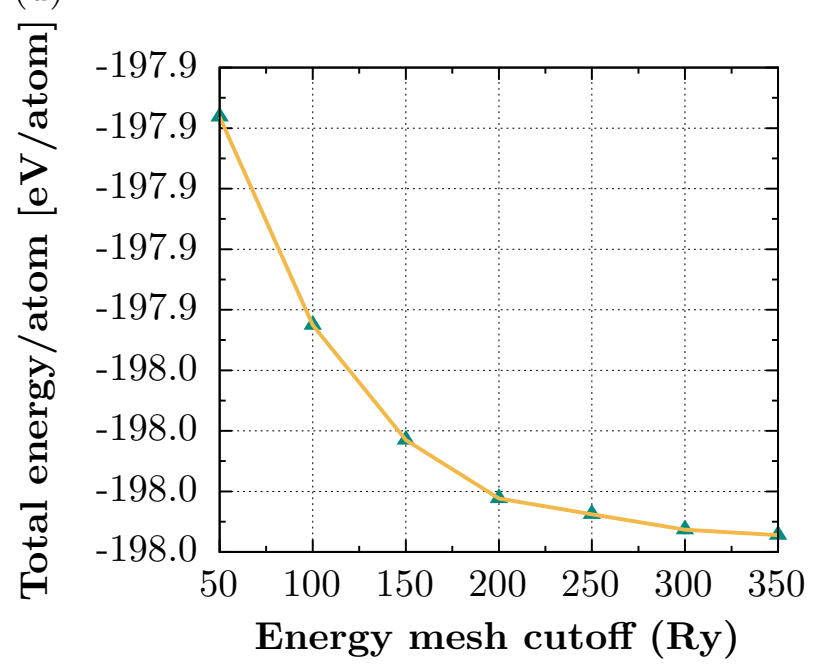

Figure 9: (a) Ground state energy for ASN as a function of the separation distance between mirror images (all directions). (b) Ground state energy for ASN for increasing mesh cutoff energy values. (c) Ground state energy for silicon nitride slab and ASN system as a function of separation distance along the z-direction between mirror images. (b) Ground state energy for silicon nitride slab and ASN for increasing mesh cutoff energy values. 
where $a_{d}=V q / d m$ and $k_{d}=6 \pi \nu \mathrm{r} / \mathrm{m}$. Here we assumed the miniprotein was a sphere of mass $m$, radius $r$ (taken to be half the length of the protein), and $\eta_{\mathrm{water}\left(25^{\circ}\right)}=0.89 \mathrm{mPa} \cdot \mathrm{s}$ is the viscosity of the medium. We integrated this differential equation to obtain the velocity and position of the protein as a function of time. We found the miniprotein experiences a short acceleration period and quickly reaches terminal velocity within the first few ps, as shown in figure 10(b). From the position as a function of time

$$
x(t)=\frac{a_{e}}{k_{d}} t+\frac{a_{e}}{k_{d}^{2}}\left(e^{-k_{d} t}-1\right),
$$

we numerically solved the dwell time based on the thickness of the membrane as shown in figure 10(c).

\section{References}

1. Sievers, F.; Wilm, A.; Dineen, D.; Gibson, T. J.; Karplus, K.; Li, W.; Lopez, R.; McWilliam, H.; Remmert, M.; Söding, J.; Thompson, J. D.; Higgins, D. G. Fast, scalable generation of high-quality protein multiple sequence alignments using Clustal Omega. Molecular Systems Biology 2011, 7, 539, Publisher: John Wiley \& Sons, Ltd.

2. Berman, H.; Henrick, K.; Nakamura, H. Announcing the worldwide Protein Data Bank. Nature Structural \& Molecular Biology 2003, 10, 980-980.

3. Koopman, E. A.; Lowe, C. P. Advantages of a Lowe-Andersen thermostat in molecular dynamics simulations. The Journal of Chemical Physics 2006, 124, 204103, _eprint: https://doi.org/10.1063/1.2198824.

4. Lowe, C. P. An alternative approach to dissipative particle dynamics. Europhysics Letters (EPL) 1999, 47, 145-151, Publisher: IOP Publishing.

5. Aksimentiev, A.; Schulten, K. Imaging $\alpha$-Hemolysin with Molecular Dynamics: Ionic 

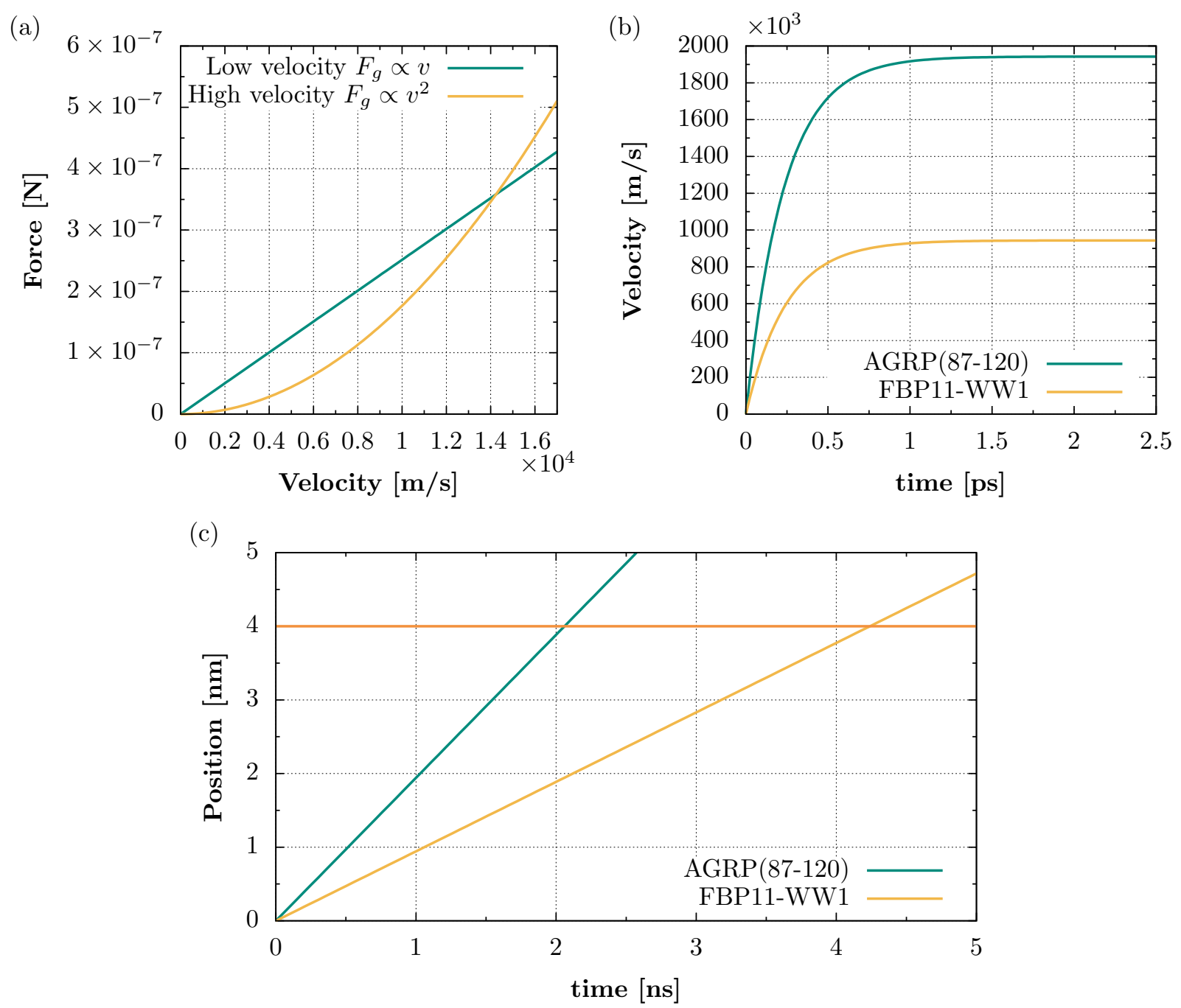

Figure 10: Determining the dwell time constant $A$ for each miniprotein based on the electric field and drag forces. (a) Approximation of the drag force from two limiting cases for large and small velocity. (b) Miniproteins reach terminal velocity within the first few ps. (c) Calculation of $A$ based on the movement of the miniproteins over time and approximate thickness of the silicon nitride membrane. 
Conductance, Osmotic Permeability, and the Electrostatic Potential Map. Biophysical Journal 2005, 88, 3745-3761, Publisher: Elsevier.

6. Coury, L. Conductance Measurements Part 1 : Theory. Current Separations 1999, 18, 91-96. 